\title{
TECNOCIENCIA Y COLONIALISMO EN LA HISTORIA DE LAS CINCHONA
}

\author{
Nicolás Cuvi \\ FLACSO Ecuador \\ ncuvi@flacso.edu.ec \\ ORCID iD: https://orcid.org/ 0000-0002-3206-5672
}

Recibido: 18 abril de 2017; Aprobado: 1 septiembre 2017

Cómo citar este artículo/Citation: Cuvi, Nicolás (2018), "Tecnociencia y colonialismo en la historia de las Cinchona", Asclepio, 70 (1): p215. https://doi.org/10.3989/asclepio.2018.08

RESUMEN: En este artículo se reflexiona sobre la ciencia y tecnología (o tecnociencia) asociadas con el colonialismo. Se usa la metáfora de "capas de colonialismo" para aludir a ideas y prácticas asociadas con la tecnociencia que son continuamente innovadas, que pueden mantenerse o reaparecer en diferentes momentos, y que estructuran el hecho colonial. Se usa como caso la apropiación de plantas de quina (Cinchona spp.) en diferentes momentos entre los siglos XVI y XX. Algunas de las capas presentadas son: apropiación material del producto natural, deslocalización y relocalización del lugar de autoridad alrededor del mismo, soterramiento de los saberes y sabedores locales, inserción de ideas sobre lo que se debe hacer con esa naturaleza, quién y cómo debe apropiarla, notransferencias de tecnología, fomento de proyectos sin mayores oportunidades, entre otras. En la historia de larga duración del colonialismo alrededor de la apropiación de las quinas, se enfatiza en capas que emergen o reemergen en momentos clave.

PALABRAS CLAVE: Quinas; Andes; capas de colonialismo; imperialismo.

\section{TECHNOSCIENCE AND COLONIALISM IN THE HISTORY OF THE CINCHONA}

ABSTRACT: This article reflects on the science and technology (or technoscience) associated with colonialism. The metaphor of "layers of colonialism" is used to refer to ideas and practices associated with technoscience that are continuously innovated, that can be maintained or reappear at different times, and that structure the colonial fact. The appropriation of quina plants (Cinchona spp.) is used as a case at different times between the 16th and 20th centuries. Some of the layers presented are: material appropriation of the natural product, dislocation and relocation of the place of authority around it, burial of local knowledge and it's owners, insertion of ideas about what to do with that nature, who and how to appropriate it, non-transfers of technology, promotion of projects without greater opportunities, among others. In the long history of colonialism around the appropriation of the quinas, emphasis is placed on layers that emerge or reemerge at key moments.

KEY WORDS: Quinas; Andes; layers of colonialism; imperialism. 


\section{INTRODUCCIÓN}

La ciencia y la tecnología, y de modo general el conocimiento, han sido consideradas artefactos fundamentales para los colonialismos e imperialismos (Brockway, 1979; Headrick, 1989; Paolo y Worboys, 1993; MacLeod, 2000; Tucker, 2000; Nieto, 2006; Seth, 2009; entre otros). Tal constatación ha llevado a considerar que la ciencia y la tecnología son partes constitutivas del colonialismo y el imperialismo. Incluso se ha considerado que, para una buena parte del mundo, "la historia de la ciencia y el imperialismo es la historia de la ciencia" (Paolo y Worboys, 1993, p. 102).

Imperialismo y colonialismo no están mediados solamente por la intervención de un poder militar: tienen que ver con el poder de modo amplio, en ámbitos semióticos y materiales. Cuando se trata de dominar o controlar las sociedades y la naturaleza, el ejercicio del poder colonial es sinérgico y aumentativo: dominar la naturaleza sirve para dominar y controlar las sociedades (Drayton, 2000), y viceversa.

Las imbricaciones entre ciencia, tecnología y colonialismo son diversas y forman parte de una compleja entidad, el hecho colonial, conformada por lo que llamo "capas". Tales capas, similares a las cortezas de un árbol, van apareciendo a lo largo de la historia, pero no sustituyen a las existentes, sino que se les añaden, aumentando la complejidad y profundidad temporal del hecho colonial. Algunas capas son de larga duración (Braudel, 1958/2007), sea porque se mantienen o porque reaparecen de manera recurrente, como fue constatado a través del análisis de la apropiación histórica de las quinas y sus alcaloides. Aún en nuestros días continúan apareciendo capas de colonialismo, en relaciones coloniales y de colonialidad que son rastreables.

En un trabajo anterior abordé la apropiación de las quinas durante la Segunda Guerra Mundial (Cuvi, 2011). En este artículo me propongo indagar si las capas detectadas en ese corto período del siglo XX han sido de mayor duración. Para ello revisé algunas fuentes primarias y secundarias de momentos emblemáticos en la apropiación de las quinas, entre los siglos $X V I$ y XX. Entre las fuentes primarias menos conocidas hasta ahora se incluyen aquellas sobre la década de 1940, archivadas en los National Archives at College Park y National Agriculture Library (Maryland), y en los New York Botanical Garden Archives.

Intentaré ilustrar que varias formas de apropiación de esas plantas, semióticas y materiales, se han mantenido o resignificado a lo largo de varios siglos. Hay patrones en el hecho colonial alrededor de las quinas, que incluyen la suplantación y asimilación de saberes sin reconocimiento de sus portadores originales, menosprecio por esos mismos habitantes, destrucción de la naturaleza, relocalización de los sitios de producción y de las formas de obtener los alcaloides, biopiratería, uso de tecnologías emergentes como las del ámbito biomolecular para nuevas relocalizaciones, construcción de modos "formales" de entender e interpretar ese mundo natural, entre otras. Esos patrones son estructurados por capas de colonialismo que, en más de un sentido, guardan estrecha relación con patrones detectados en investigaciones sobre colonialidad del saber, del ser, del poder y de la naturaleza en América Latina (Quijano, 2000; Castro-Gómez y Grosfoguel, 2007; Alimonda, 2011; entre otros).

Entre los efectos de muchas de esas capas de colonialismo se cuenta el que los nativos o locales de los territorios coloniales y poscoloniales piensen que carecen de conocimientos, o que los que poseen son inútiles a la luz de la modernidad. Al mismo tiempo, muchos creen que podrán acceder a esos conocimientos traducidos a nuevos cánones (Seth, 2009). Capas de colonialismo se han insertado en los pensamientos, subjetividades, cuerpos, territorios y recursos, con la tecnociencia actuando de modo clave para ello, disciplinando el conocimiento, dotándolo de objetividad y razón, reformulando las preguntas, métodos y objetivos, trayendo a la escena máquinas, mapas, películas, artículos, libros, conferencias, ideas sobre la naturaleza, su propósito y las formas de apropiarla.

Hoy parece normal reflexionar sobre la ciencia y la tecnología como aliadas fundamentales de la empresa colonial, útil para dominar los territorios coloniales y sus sociedades, con o sin ocupación militar. La colonialidad ha sucedido en casi todo el mundo en diferentes momentos desde el siglo XV y sus capas se han mantenido en ciertos territorios poscoloniales del siglo XXI; todavía la ciencia y la tecnología son cruciales para reinventar, reconstruir, reorganizar y consolidar relaciones hegemónicas y dispares ilustradoras del colonialismo. Como aporte a esas ideas, con este artículo pretendo dos cosas: introducir la idea/metáfora de capas de colonialismo para interpretar las partes semióticas y materiales que estructuran el hecho colonial; y ampliar el alcance de carácter explicativo de unas fuentes que, a propósito de ciencia e imperialismo, analizo desde hace algunos años (Cuvi, 2009).

Por supuesto, no quiero decir que las capas aquí explicadas sean exclusivas de la esfera de las quinas; sin duda aparecen también en la apropiación de otros productos naturales, domesticados y silvestres, y de las 
sociedades. En el marco de la reflexión sobre los complejos modos mediante los cuales circulan conocimiento y poder, especialmente en las relaciones Norte-Sur, o imperio-colonia, en el artículo también intento dar cuenta (en ocasiones desde los silencios, en ocasiones de modo tangencial, en otras a partir de fuentes primarias) del papel de ciertos actores que han sido soterrados y que han sido fundamentales en los procesos de estructuración del hecho colonial. Esa reflexión sobre la participación local en la construcción y circulación de saberes, en los procesos de excelencia, en las resistencias, en la pervivencia de conocimientos y su reemergencia, ha sido abordada por varios autores (Cueto, 1995; Castro-Gómez, 2005; Cañizares-Esguerra, 2006; Gorbach y López Beltrán, 2008; Herrera y Ali, 2009; Fernández Prieto, 2015a, 2015b; entre otros).

La invisibilización del otro es parte de la construcción del imperialismo, como ha sido mostrado para registros visuales como pinturas (Bleichmar, 2009). Asimismo, varios trabajos evidencian la existencia histórica de "saberes híbridos" y procesos de "descentramiento" y "relocalización" de la ciencia (Raj, 2007; Fernández Prieto, 2013, 2015b). Se ha sugerido que la misma llustración y modernidad han ocurrido gracias al hecho colonial y lo que este aportó al conocimiento y las sociedades (Castro-Gómez, 2005). Esos trabajos y otros afines ilustran que el hecho colonial emerge de una relación bidireccional, de ida y vuelta, con tensiones o rupturas o negociaciones o asimilaciones entre lo local y lo global, entre los centros y las periferias construidas. En cierto modo, ratifican que el papel del imperialismo y el colonialismo en la historia está lejos de ser un tema agotado. Por el contrario, dadas las capas de colonialismo que perviven en el siglo XXI alrededor de la circulación de ciencia y tecnología, parece pertinente seguir esa línea, en las indagaciones históricas y en los estudios sociales de la ciencia. No parece posible entender estructuras y procesos contemporáneos sin análisis de larga duración, que ilustren patrones y procesos históricos que, en buena medida, han condicionado los desarrollos locales, las resistencias, las hibridaciones, las agencias de todos los actores, incluso aquellos no humanos, en este caso las quinas.

El artículo continúa con una breve explicación de las quinas, seguida del análisis de algunas capas que aparecieron en diferentes momentos de su apropiación, con actores como los jesuitas de Loja al inicio del siglo XVI, La Condamine, Mutis, Ruiz y Pavón en el siglo XVIII, los contrabandistas de quinas del siglo XIX, la última gran apropiación de las quinas andinas durante la Segunda Guerra Mundial y el paso al sintético. Se termina con unas reflexiones sobre las características de las capas de colonialismo alrededor de las quinas, y lo que podrían decirnos sobre procesos más amplios alrededor de la apropiación del mundo natural.

\section{LAS QUINAS}

"Quina" es el nombre más común que reciben actualmente todas las plantas del género Cinchona y unas pocas de los géneros Remijia y Ladenbergia cuyas cortezas tienen propiedades medicinales entre las que destaca su poder antimalárico. Otro nombre muy común es "cascarilla". El poder de las quinas para prevenir y curar la malaria se debe a cuatro alcaloides presentes en sus cortezas: cinchonina, cinchonidina, quinidina y quinina, siendo el último el más importante antimalárico. Cada especie tiene diferentes concentraciones de alcaloides, pero el contenido de estos puede variar incluso dentro de la misma especie según la localidad, altitud, tipo de suelo, edad del árbol y época de cosecha. Tradicionalmente, se ha preferido aquellas especies con más porcentaje de quinina; sin embargo, la mezcla de los cuatro alcaloides, conocida como totaquina, fue usada, sobre todo por el imperio británico, durante casi 200 años.

Durante cuatro siglos de uso terapéutico esas plantas han recibido cientos de nombres científicos y comunes, creando una exasperante confusión entre naturalistas, comerciantes, médicos e historiadores. La historia refleja desde riñas científicas y comerciales hasta la incapacidad de encajar la biología de Cinchona en la nomenclatura lineana, pues sus especies tienen un elevado grado de hibridación, con cientos de subespecies, variedades, formas, y especies polimórficas (Camp, 1949). Aunque persiste la polémica sobre cuántas especies existen, en la revisión más reciente se reconocen 23 (Andersson, 1998).

Sobre las quinas hay biografías, estudios críticos y abundantes fuentes primarias y secundarias. Ha sido un tema o materia bastante visitado desde hace siglos por médicos, exploradores de esas plantas, botánicos, historiadores, historiadores de la ciencia, inclusive novelistas. Como introducciones ineludibles se citan: Wellcome Historical Medical Museum (1930), Haggis (1941), Guerra (1977), Brockway (1979), Jarcho (1993), Ortiz (1994), y Philip (1995). Aún así, esas plantas continúan ofreciendo posibilidades de exploración como la ensayada aquí.

Muchas narraciones sobre el uso de los alcaloides de las quinas, desde hace siglos, parecen insinuar un triunfo de la humanidad. Es verdad que los antimaláricos naturales y sintéticos han curado a muchas per- 
sonas (incluido el autor de este artículo), pero en contraposición aparece el vergonzoso fracaso que implica el que, pese a contar con la cura desde hace 400 años, solo se ha conseguido erradicar la devastadora malaria de algunos lugares, y hasta parece acentuada la segregación de quienes la padecen. Desde su introducción en terapéutica, los polvos de la corteza de quina, sus alcaloides y los medicamentos derivados de ese conocimiento han curado especialmente a los ricos del mundo; en el siglo XXI la malaria es considerada una enfermedad asociada con la pobreza (Worrall, Basu y Hanson, 2005). Ese primer indicio sirve para introducir a otras capas de colonialismo, de un sistema que distribuye de manera desigual y deliberada las cargas y los beneficios, los méritos y las responsabilidades, durante la apropiación del mundo natural.

\section{PRIMERAS APROPIACIONES DE QUINAS Y SABERES}

Una polémica en torno a las quinas, desde hace siglos, ha girado alrededor de si los indígenas conocían o no sus propiedades febrífugas. La evidencia presentada por Ortiz (1994) ha deducido que la planta medicinal fue documentada en el siglo XVI por Nicolás Monardes y Juan Fragoso, protobotánicos dedicados a compilar informaciones sobre los productos naturales que llegaban a Europa desde Oriente y Occidente. Según ese autor "hay base para afirmar que la Quina fue probablemente conocida como materia médica desde mucho antes de su "descubrimiento" en la primera mitad del siglo XVII. Este conocimiento seguramente tuvo origen en los indígenas, quienes [...] usaban la corteza con ventaja y la compartieron enseguida con los españoles" (Ortiz, 1994).

Fragoso y Monardes no dieron nombre a las plantas medicinales en sus textos de 1572 y 1574, respectivamente, pero conforme los polvos de quina se popularizaron en la terapéutica europea, a partir de 1630, se les otorgaron varios. Uno de ellos, chinchona, proviene de una historia propia del realismo mágico y que tuvo similar éxito comercial: se corrió la voz de que la Condesa de Chinchón, Virreina del Perú, se curó de la malaria usando polvos de quina que le proveyeron los jesuitas de Loja. Aliviada de su dolencia, habría entregado de forma altruista dichos polvos amargos a la sociedad limeña para que nadie continuara padeciendo la enfermedad. Esa historia con tintes nobiliarios fue reproducida por eminencias científicas durante 300 años, cuestionada desde el siglo XIX, pero solamente en 1941 se probó su falsedad: no existió tal curación de la condesa ni los polvos de quina circularon entre los pobres de Lima (Haggis, 1941). Pero la fábula cumplió el propósito de quien la inventó: legitimar el uso de los polvos de quina entre la nobleza, contando con una autoridad (de la realeza y de los jesuitas) para popularizar el producto. Otros nombres que se han dado a las quinas son "China China” (en italiano) y "Quinquina".

Desde varias narrativas se aseguró que los indígenas no conocían las propiedades medicinales de las quinas y que ellas fueron descubiertas por los jesuitas. Esos filtros de autoridad iban descentrando el conocimiento, pues si bien es posible que los jesuitas hayan usado la corteza para tratar la malaria, quedan dudas de que hayan sido los descubridores de sus propiedades medicinales. En estos ejemplos se detectan capas de colonialismo.

La quina penetró muy bien la terapéutica, no sin polémicas (Jarcho, 1993). Llegaban cortezas y polvos a Europa para prevenir y curar la malaria, pero durante más de 100 años se desconocía cómo eran los árboles de los que eran extraídos. En parte para llenar ese vacío, dos miembros de la Expedición Geodésica HispanoFrancesa a la Provincia de Quito, los franceses Joseph de Jussieu y Charles Marie de La Condamine, se encargaron de inscribir las plantas en el terreno de la botánica taxonómica, al realizar su descripción morfológica. Motivado por la ansiedad ilustrada de describir el mundo natural y apropiarse del mismo, llevar sus partes a los centros de conocimiento para describirlas a la luz de un museo, y con la intención de contrabandear semillas para establecer plantaciones en otros territorios y romper el monopolio de las colonias españolas, La Condamine hizo una exploración de las quinas de Loja en 1737. Él era geógrafo -estaba en América para determinar la forma de la Tierra y medirla-, pero aprovechó un paso por Loja para describir las quinas de la región lo mejor que pudo, gracias a algunas instrucciones básicas del botánico Joseph de Jussieu. La Condamine recorrió los bosques de Cajanuma guiado por indígenas y mestizos expertos en la cosecha y preparación de Cinchona, tras lo cual publicó su Estudio sobre la quina (de La Condamine, 1778/1986) que envió al sueco Linnaeus, quien llamó a la planta Cinchona officinalis en referencia a la leyenda de la Condesa de Chinchón. Jussieu hizo una extensa obra en 1737 sobre esas plantas, pero solo fue ampliamente conocida siglos después (Jussieu, 1737/1936), con lo cual el crédito por arrojar luz sobre la planta fue para La Condamine.

La Condamine no pudo ser el primero en contrabandear las semillas a Europa (capa de colonialismo que explicaré más adelante), pues perdió su cargamento durante su viaje por el Amazonas en 1743 (de La Condamine, 1745/1986). Pero hizo la primera descripción botánica, construyendo un conocimiento sobre la planta que aumentaba las posibilidades de 
controlarla, que la llevaba hacia otros centros e iba resignificando ese control. Él, Linnaeus y otros ilustrados europeos fueron insertando a las quinas en un modelo que fue convirtiéndose en hegemónico para nombrar y clasificar el mundo natural. El conocimiento y la autoridad pasaban cada vez más del dominio de los sabedores y cascarilleros locales al de los botánicos europeos, con sus herbarios, libros de texto y academias. La Condamine describió a las plantas por sus colores y propiedades, siguiendo las normas de los sabedores locales, pero cuando pasó al mundo de la botánica esas ideas se fueron perdiendo en favor de un canon que excluía tradicionales formas de clasificación del mundo natural. La imposición de lenguajes y formas de clasificar la flora fue una práctica común en los Andes y otros lugares; ocurrió por ejemplo con los resultados de la expedición botánica de Francisco Hernández hacia el Virreinato de Nueva España, cuando el médico napolitano Nardo Antonio Recchi fue incapaz de "entender la ordenación original de la obra, percibiendo las aportaciones innovadoras de Hernández y los criterios taxonómicos indígenas que este había aprovechado" (López Piñero y Pardo Tomás, 1994, p. 70). Al nombrar y hacer encajar las plantas en un sistema se construían nuevas capas de colonialismo.

La ciencia colonial fue suprimiendo y suplantando el conocimiento del otro sobre la naturaleza; lo tradujo y reclamó un nuevo centro de autoridad para el saber. $Y$ con ello fue reforzando ideas sobre quiénes pueden conocer, qué y cómo, y las formas de expresarlo. En los encuentros del colonialismo con las propiedades medicinales y las características botánicas de las quinas se fueron ocultando y transformando los sabios y sabedores locales, desplazando el "centro" de ese conocimiento, sin que ello supusiera que los saberes hayan desaparecido.

Poco después de Charles de La Condamine aparecieron otros ilustrados deseosos de conocer y apropiar el mundo natural. Asociado con un interés comercial, en el siglo XVIII se enviaron dos expediciones botánicas a los virreinatos andinos para mejorar el conocimiento sobre las quinas. El flujo de corteza hacia la Real Botica en ese siglo se había incrementado por lo que se estableció un estanco, usando como justificaciones principales las constantes adulteraciones y la sobreexplotación de los bosques. Como no había análisis químicos para determinar la cantidad de quinina (solo conseguidos hacia 1820 por Joseph B. Caventou y Pierre J. Pelletier), ocurrían muchas falsificaciones. Crawford (2007) ha explicado cómo en ese contexto la Corona española fue construyendo -o reforzando- una idea de que las personas involucradas en la extracción, comercio y transporte de la corteza de quinas eran ignorantes, carentes del conocimiento científico que estaba en manos de boticarios y botánicos. Durante los 38 años que duró el estanco (1751-1790), se enviaron 350 mil libras de corteza de quina hacia la Real Botica (de Andrés Turrión, 1989).

En tiempos del estanco se planificaron dos expediciones botánicas para la apropiación de productos naturales de los Andes, especialmente quinas, lideradas por Hipólito Ruiz y Josef Pavón al Virreinato del Perú desde 1777, y por José Celestino Mutis al Virreinato de Nueva Granada desde 1783. Hubo una competencia entre ambas por determinar cuál territorio contenía las mejores quinas (Ruiz, 1792/1994; Mutis, 1828/1994) ${ }^{1}$. Sobre la expedición a Nueva Granada, Nieto Olarte (2006) ha explicado que en ese proyecto de inventario del mundo la historia natural y la política deben ser consideradas expresiones de la misma estructura de poder. Esas exploraciones estaban embebidas en la tradición de dominar la fuerza de trabajo y la naturaleza mediante viajes y acumulación de información en forma de especímenes de herbario, notas de campo, mapas, dibujos, semillas, plantas, que permitieran construir un conocimiento hegemónico (Krige, 2006).

En cuanto al reconocimiento del papel de los actores locales, Hipólito Ruiz reconocía que "no se le puede negar al Indio el mérito de la noticia dada" sobre las propiedades medicinales de las quinas (Ruiz, $1792 / 1994)$, pero no queda claro hasta qué punto un crédito de "noticia" puede aludir a uno de "saber". De todos modos, aquello fue, en contexto, una enorme concesión, dado que Ruiz se encargó de desprestigiar cualquier valor atribuible a los nativos y sus conocimientos, llamándolos belicosos, perezosos, supersticiosos. Como muchos otros "cazadores" de quinas, Ruiz alertó sobre la destrucción de los quinares y la necesidad de repoblar con esas especies, asunto que está en consonancia con las políticas forestales de aquellos tiempos, suscitadas por los efectos del esquilme de las tierras ibéricas (Urteaga, 1987).

También Mutis hizo anotaciones sobre la ignorancia de la sociedad que lo rodeaba (Bleichmar, 2009), con lo cual la desvalorización, soterramiento, descentramiento de saberes, continuó (re)emergiendo ligado a la historia de las quinas. Algunos años más tarde, el prusiano Alexander von Humboldt puso en duda que los indígenas hayan conocido las propiedades de la corteza, reiterando que fue por la experimentación de los misioneros jesuitas que se comenzaron a usar las infusiones de corteza: 
This tradition is less improbable than the assertion of European authors, and among them the late writers Ruiz and Pavon, who ascribe the discovery to the Indians. The medicinal powers of the Cinchona was likewise entirely unknown to the inhabitants of the kingdom of New Granada (Humboldt, 1821, p. 23).

\section{RELOCALIZACIÓN DE LOS SITIOS DE PRODUCCIÓN EN EL SIGLO XIX}

La explotación de las quinas ocasionó estragos que fueron advertidos por varias personas a fines del siglo XVIII. El criollo Eugenio Espejo (1792) sugirió reforestar urgentemente con quinas y otro criollo, Francisco José de Caldas (1805/1966), se quejaba de la destrucción en Loja y que no se habían plantado árboles. Pero poco o nada se hizo, lo cual aumentó las voces en la primera mitad del siglo XIX, sobre todo en Europa, que mencionaban que esas plantas estaban al borde de la extinción. Esa situación, junto con el deseo de controlar el comercio de corteza de quina y de autosuficiencia en la producción de quinina, motivó que se insistiera con cada vez mayor intensidad en la necesidad de contrabandear semillas para llevarlas a las montañas de las colonias asiáticas -con características ambientales bastante similares- y romper con el monopolio que había sido de España y de las repúblicas andinas. Habían aumentado los volúmenes exportados, en buena medida por la demanda de quinina del imperio británico, convertida en un instrumento fundamental para la penetración en territorios tropicales (Headrick, 1989). Al mismo tiempo, la polémica desatada en torno a la calidad de las quinas de Nueva Granada y del Perú estaba siendo dejada de lado a partir del aislamiento de la quinina en 1820 (Pelletier y Caventou, 1821). Desde esa fecha el laboratorio se sumó como un nuevo espacio para el control de los alcaloides de las quinas. Con los análisis precisos, las quinas de Loja perdieron protagonismo en favor de las de Bolivia y Colombia, aumentando la frontera extractiva por el incremento de la demanda.

Varias expediciones fueron enviadas en el siglo XIX con el fin de contrabandear semillas (Markham, 1862; Wellcome Historical Medical Museum, 1930; Brockway, 1979). Esa acción de relocalización territorial del producto, que ya había sido intentada con las quinas desde La Condamine, materializó una nueva capa de colonialismo, que se sumaba a otra de mayor profundidad temporal, relacionada con la construcción de la idea de la incapacidad de las repúblicas andinas para gestionar el recurso.

Los gobiernos de Inglaterra y Holanda (de manera independiente) se plantearon al más alto nivel políti- co contrabandear semillas de quina de los Andes. Tras sucesivos intentos perpetrados en Bolivia, Perú, Ecuador y Colombia, el primer contrabando exitoso fue conseguido desde el Ecuador (Spruce, 1860/1996). En una carta Richard Spruce escribió sobre la importancia que tuvo para su trabajo un cascarillero de apellido Bermeo (Spruce, 1859/1996). Semillas de C. pubescens fueron llevadas a Kew Gardens y al sureste asiático (Brockway, 1979). Así fue como un recurso que había pasado por un control colonial y luego poscolonial, regresaba a uno colonial gracias a la relocalización territorial. El imperio británico ganó la carrera del espionaje, pero fueron los holandeses quienes tuvieron mayor éxito en las plantaciones.

En 1865, el inglés Charles Ledger consiguió que el indígena Manuel Incra Mamani contrabandeara de Bolivia semillas de $C$. calisaya, la especie silvestre con mayores concentraciones de quinina. El gobierno holandés le pagó una pírrica suma que en nada cubría la inversión realizada, menos aún la vida de Mamani, torturado por las autoridades bolivianas por contrabandear las semillas (Gramiccia, 1988). Otro testimonio del papel que tuvieron los sabedores locales. La C. calisaya fue sembrada en Java y algunas décadas después, gracias a la aplicación de técnicas de forestería científica, se consiguieron árboles que duplicaron la cantidad de alcaloides de los especímenes silvestres. La mejora fue de tal magnitud que se creó la que es reconocida en la nomenclatura contemporánea como nueva especie, $C$. ledgeriana, nombrada en homenaje a Ledger, no a Mamani. Emergió un monopolio holandés sobre la producción de corteza y su manufactura, al tiempo que la producción andina se estancó. Salían de los Andes pequeños cargamentos con poca influencia en el mercado mundial y que los mismos comerciantes holandeses compraban para sacar del mercado. Bolivia fue la república que intentó mantener cierta participación en los mercados, promovió plantaciones de cientos de miles de árboles, pero resultaban insuficientes y padecieron extrañas plagas que incluso se adujeron al sabotaje (Pardo Valle, 1947).

La desterritorialización de los árboles de quina, una capa de colonialismo, fue determinante para la geopolítica alrededor de las plantas y sus alcaloides hasta la década de 1940. Contribuyó a descentrar aún más los saberes, a fortalecer imaginarios de que los pobladores de los Andes eran incapaces de controlar su producción. Ya no se trataba solamente de soterrar a los indígenas, sino a los criollos y a las repúblicas andinas. 


\section{PLANTACIONES SIN FUTURO, TECNOLOGÍA NO TRANS- FERIDA Y EL PASO AL SINTÉTICO EN EL SIGLO XX}

Estados Unidos era un gran consumidor de quinina e intentó, especialmente desde la década de 1920, acabar con el monopolio holandés mediante varias estrategias: búsqueda de sustitutos naturales, intentos de síntesis artificial de quinina, fomento de plantaciones y lobby internacional. Los alemanes también fueron muy activos en el desarrollo de sintéticos ${ }^{2}$. Sin embargo, solo se transformó la dinámica durante la Segunda Guerra Mundial, cuando Estados Unidos requirió apertrecharse de materias primas estratégicas. Los envíos de quinina desde el sureste asiático habían cesado por la invasión japonesa, por lo que Estados Unidos puso en marcha una dinámica maquinaria institucional, apoyada en lo tecnocientífico, para reactivar la extracción de quina de los Andes. Creó varias agencias que fueron aglutinadas bajo el paraguas del Cinchona Program, la mayor prospección jamás hecha de una sola planta medicinal en la historia de la humanidad (Cuvi, 2009; 2011). Decenas de botánicos, agrónomos, químicos y forestales (apoyados por una enorme maquinaria institucional) prospectaron las quinas y promovieron su extracción, producción, y exportación hacia fábricas de los Estados Unidos.

La guerra fue aprovechada para apropiar corteza de quina y muchos otros productos. En 1940 se hicieron inventarios en busca de oro, manganeso, cromo, bauxita, estaño y otros minerales, patrocinados por el Interdepartmental Committee on Cooperation with the American Republics. Se hizo estudios de los recursos pesqueros de Perú y todos los países caribeños. Hubo misiones a México y Honduras para investigar la posibilidad de producir vegetales de interés para Estados Unidos. Brasil, aliado estratégico en el tema caucho, recibió en el verano de 1942 a la American Technical Commission, enviada para planificar el desarrollo del transporte, energía, minas, industrias, etc., que favorecerían el comercio con Estados Unidos. Muy relevantes fueron las misiones forestales, inventarios de recursos agrícolas y forestales en busca de todas las plantas económicas. Para 1944 el número de misiones relacionadas con el desarrollo de recursos naturales en América era "too large to permit complete listing"

Volviendo a las Cinchona, se fomentaron viveros y plantaciones desde México hasta Bolivia. En la finca guatemalteca "El Porvenir" se desarrolló el mayor vivero de una planta medicinal en el mundo (Rosengarten, 1944). La búsqueda de antimaláricos cruciales para la guerra también llevó a que se ejecutaran investigaciones de laboratorio y ensayos clínicos en escalas nunca antes probadas. Esas investigaciones tuvieron éxito, en algunos casos a partir de moléculas que habían sido desarrolladas décadas antes pero no habían sido suficientemente probadas (Wiselogle, 1946). Tales programas de investigación en biomedicina, junto con el Cinchona Program, por su magnitud, pueden ser entendidos como episodios de Gran Ciencia en biología y biomedicina, que podemos considerar como otra capa de colonialismo, una que lleva a crear un "encantamiento del crecimiento", una ilusión sobre posibilidades de crecimiento a velocidades y escalas que parecen inalcanzables para ciertas sociedades del Sur, por ejemplo por las diferencias de capacidades instaladas (Cuvi, 2012).

La necesidad de prevenir y curar la malaria llevó a recuperar, desarrollar y probar medicinas sintéticas, y a que las quinas silvestres y las viejas y nuevas plantaciones americanas volvieran a ser estratégicas. Estados Unidos intentó, en algún momento al principio, relocalizar hacia su territorio nacional las quinas, sin éxito por las condiciones climáticas. Entonces distribuyó plántulas de quina por todo el continente para fomentar plantaciones; algunas de las semillas provenían de Filipinas, sacadas in extremis antes de la ocupación japonesa. Pero al final de la guerra las plantaciones por toda América fueron olvidadas, incapaces de competir con las asiáticas, donde la calidad era mayor y la mano de obra más barata. Tal fracaso no fue un imprevisto para el gobierno estadounidense, que ocultó información a las repúblicas americanas. Por ejemplo, en un informe en el cual se delinearon estrategias de colaboración con el Ecuador en torno a varios productos, sobre todo quinas y caucho, en el prefacio se aclaraba entre paréntesis: "The foreword should be excluded from the report submitted to the Ecuadorean Government"4 . Se controlaba la planificación -qué hacer, cómo hacerlo- sin entregar toda la información, pero haciendo pensar a las repúblicas que era lo adecuado. Desde una posición de autoridad, tecnocientífica, militar, económica, se construían imágenes falsas, inventando, recuperando o resignificando capas de colonialismo.

Otra capa de colonialismo emergió en el marco de los procesos de (no) transferencia de tecnología. En los acuerdos bilaterales que Estados Unidos hizo con los países andinos a comienzos de la década de 1940 se especificaba que algunas cortezas serían procesadas a nivel local, para lo cual Estados Unidos transferiría tecnología de punta a las industrias existentes y en algunos casos financiaría una nueva fábrica ${ }^{5}$. Pero fue obviado y la asistencia se concentró en las planta- 
ciones (que tenían futuro si Estados Unidos ganaba la guerra). Impulsar industrias en los Andes no concordaba con la política de comprar materias primas y vender procesados, la política de la complementariedad. En las reuniones de las agencias de guerra económica se mencionaba que establecer fábricas en América Latina sería promover la competencia con lo doméstico, por lo cual la decisión de no enviar tecnología prevaleció sobre cualquier acuerdo. La War Production Board estaba "definetely committed to a policy of preventing us from assisting Latin American countries in setting up extraction plants" ${ }^{\prime \prime}$. Al tiempo que se apropiaban las cortezas y los conocimientos alrededor de ellas (viejas capas), se impedía que los conocimientos y réditos de su extracción fuesen transferidos a los Andes.

También la invisibilización de los sabedores reemergió durante ese momento de apropiación del producto. Pese al declive de décadas del mercado andino, mucho conocimiento sobre las quinas había pervivido entre curanderos y chamanes. Algunos de esos conocedores, los "cascarilleros", fueron contratados como asistentes de campo y enseñaron a los científicos cómo moverse, dónde hacerlo, reconocer plantas, etc. Pero ello fue negado por la mayoría de técnicos estadounidenses, sobre todo mediante la omisión, el silencio, pero también de manera explícita, como cuando Walter Hodge, jefe de la Misión de Cinchona en el Perú (que era parte del Cinchona Program), aseguró que los cascarilleros habían muerto una generación antes de las misiones y que los botánicos debían enseñar a los asistentes cuáles árboles eran Cinchona (Hodge, 1948). Ross E. Moore, subdirector de la Office of Foreign Agricultural Relations (OFAR), reconoció que los trabajos eran como un "entrenamiento" (Henningson, 1981) y que los científicos enviados sabían poco, pero tampoco reconoció el papel de los saberes locales. Sin embargo, las fuentes de Wendell H. Camp desmienten esas aseveraciones y revelan cuestiones detrás de los silencios. Él reconoció la importancia de los sabedores locales. A diferencia de otros expedicionarios, contemporáneos y anteriores, se encargó de perpetuar su mérito. Uno fue Francisco Prieto (Figura 1 ), indígena proveniente de un linaje de quineros que conocía bien las cortezas del sur del Ecuador y que guió a todos los exploradores estadounidenses que fueron allí antes y durante la Misión de Cinchona al Ecuador. Prieto era tan indispensable que llegó a ser contratado por la Corporación Ecuatoriana de Fomento. Camp recordó que Prieto:

Took pains to teach me the lore of cascarilla bark hunting, as well as the multitudinous ways in which the bark was faked or diluted. He was a conscientious and excellent workman and completely to be trusted to operate alone. As a result he was often sent on special trips when it was no expedient to make up a fullscale expedition (Camp, 1952).

El botánico le enseñó a colectar muestras y llevar un cuaderno de campo, en el cual Prieto mezclaba casteIlano con quichua. Algunas muestras que aún reposan en herbarios de los Estados Unidos llevan el nombre de Prieto, lo cual parece compensar los problemas que Camp tuvo por ello, pues al querer dar crédito a sus asistentes complicó la sistematización de información. Por ello, sin embargo, han pasado a constar merecidamente entre los coleccionistas botánicos del Ecuador (Balslev y Joyal, 1980).

Los cascarilleros y otros asistentes se encargaban desde abrir caminos hasta verificar informaciones. Cuando los indígenas venían a mostrarle cascarilla a Camp, este enviaba a Prieto para aprender el camino ${ }^{7}$. Esos asistentes estaban dispuestos a todo, incluso contrabandear objetos para Camp, en otro paralelismo que recuerda la capa asociada con la historia de Ledger y Mamani, aunque a diferencia de las autoridades bolivianas, el mismo Director Nacional Forestal, Misael Acosta Solís, ofreció ayuda a Camp ${ }^{8}$.

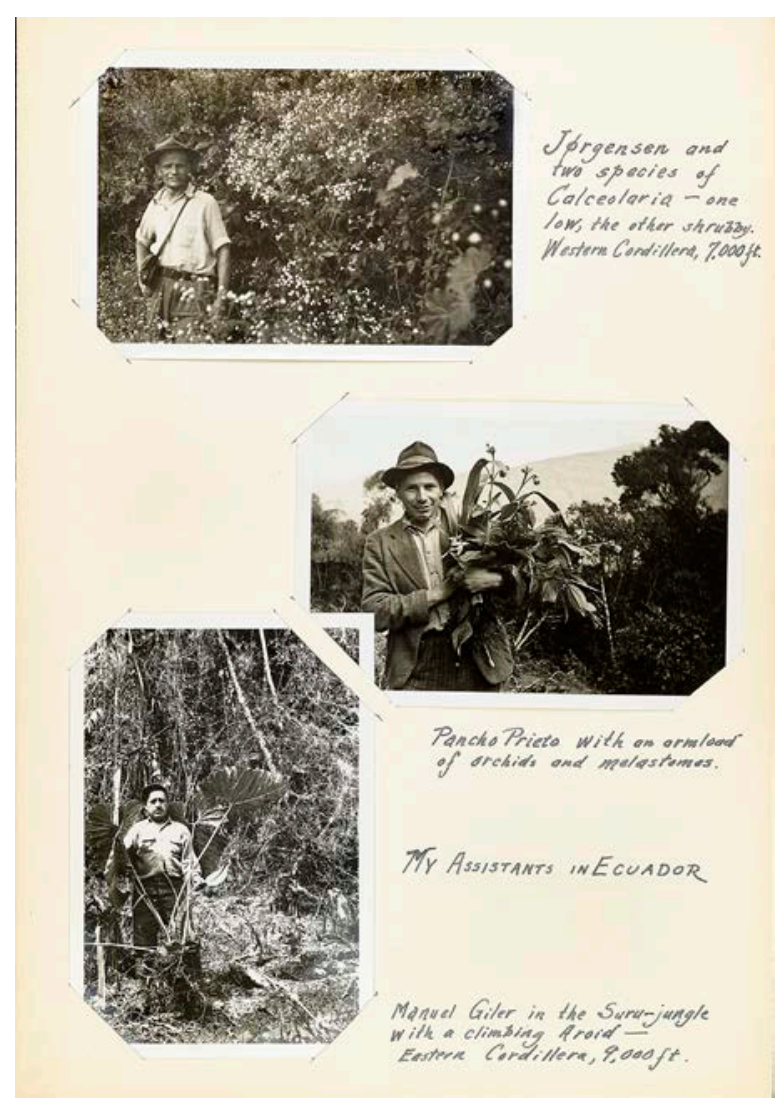


Al respecto de Camp conviene añadir que, al igual que La Condamine, clasificó a las quinas recurriendo tanto a nombres locales como a la clasificación botánica. Eso se observa en sus mapas de campo, donde menciona los nombres comunes. En la Figura 2 alude hasta a 12 posibles variedades dentro de por lo menos 5 especies. Al igual que sus predecesores exploradores de las quinas, Camp fue exasperado por la esquiva clasificación de Cinchona y mantuvo una polémica con otro miembro de la Misión de Cinchona, Raymond Fosberg, sobre este asunto. Intentó resolver el asunto basado en la cantidad y tipo de alcaloides, que ilustraban la variación entre áreas e individuos de diferentes grupos (Camp, 1949), algo pionero al considerar rasgos bioquímicos para la taxonomía.

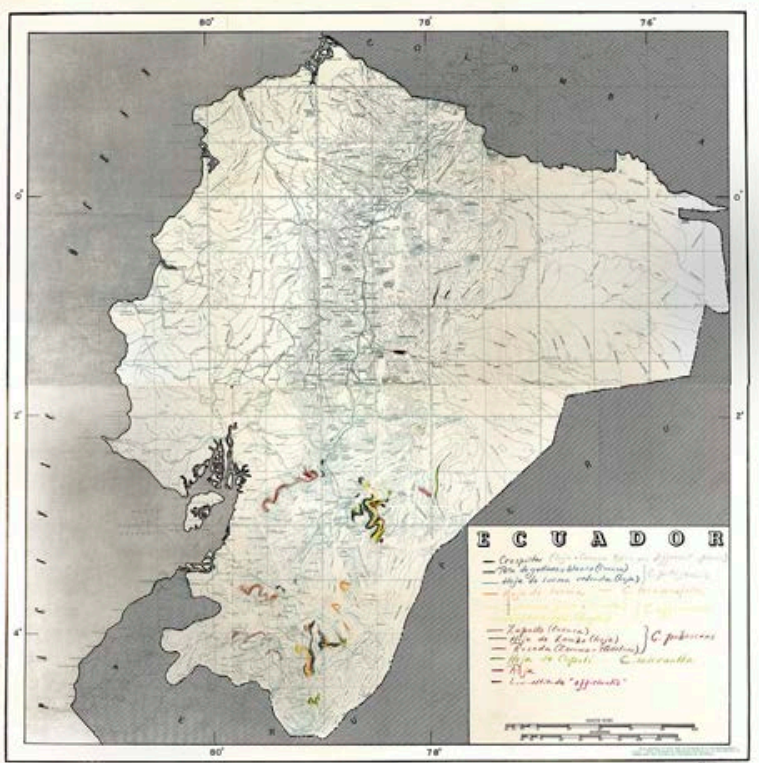

Otra capa de colonialismo que reapareció en esa época está relacionada con las opiniones sobre las sociedades locales. El botánico Ira Wiggins (también de la Misión de Cinchona) expresó su deseo de "volver a la tierra de los civilizados" y cuando lo hizo, al referirse a los científicos latinoamericanos que estaban siendo becados a los Estados Unidos, dudaba de sus posibilidades de aprovechar esas becas, aduciendo que preferían pasar la vida en bares 9 . Un alto cargo de la Foreign Economic Administration, Claude Courand, envió a fines de 1944 una serie de características sobre la forma de vida en el Ecuador, asociándola con estructuras coloniales y al mismo tiempo reproduciéndolas: "1. Life in Ecuador still partakes of much of the colonial era. Where modern conveniences are available, they rarely function; 2 . The population is nearly $90 \%$ Indian and rubbing elbows with them is inevitable ${ }^{\prime 10}$. Sobre ese país, otro consultor dijo que "there are possibly 2000 who are competent by position, familiy, education and experience to express a qualified opinion on subjetcs of national importance"11. Se construía la idea de que apenas se podía hablar con dos mil personas en una población de millones, ignorantes de cómo gestionar su territorio.

Finalmente, en la segunda mitad del siglo XX el desarrollo de los antimaláricos sintéticos supuso una nueva deslocalización de las quinas, aún más hacia el laboratorio, al pasar las curas de la malaria al control de industrias farmacéuticas, protegidas por dispositivos como patentes, novedosas formas de señalar quién es el dueño del conocimiento y las formas para apropiarlo. Uno de los últimos hitos en la apropiación de las quinas en el laboratorio fue la síntesis artificial de la molécula de quinina. Una síntesis muy sonada fue la de los estadounidenses Robert Burns Woodward y William von Doering (1944), quienes obtuvieron homomeroquinina, importante precursor de la quinina. Pero tuvieron que pasar varias décadas, hasta 2001, para que un equipo lograra la síntesis total de la quinina en su correcto isómero (Stork et al., 2001).

Del control de los jesuitas se fue pasando a la botánica, las boticas, la ciencia colonial holandesa, el colonialismo estadounidense y las grandes industrias farmacéuticas. Una capa que ha pervivido es la biopiratería (antes contrabando), que consiste en obtener conocimientos de productos naturales y llevarlos a laboratorios donde son analizados y patentados. Esos dispositivos reflejan capas de colonialismo asociadas con quién conoce y cómo hacerlo, en una historia (la del colonialismo) que es de larga duración.

\section{REFLEXIONES FINALES}

La historia de la apropiación de las quinas en el marco de imperialismos y colonialismos, ilustra algunas formas mediantes las cuales los imperios han impuesto más que negociado sus técnicas e ideas, para construir paisajes y sociedades acordes con sus intereses y visiones de productividad (Osborne, 2000). En esos procesos, la tecnociencia se ha desplegado a través de dispositivos semióticos y materiales, cuya estructura puede ser comparada con las capas de un árbol, el hecho colonial.

Las capas de tecnociencia y colonialismo han aparecido en diferentes momentos, a veces reaparecido, a veces han estado presentes de manera continua. En las quinas se manifiestan alrededor de la agricultura, medicina, ingenierías, botánica y química, siempre con el apoyo de instancias políticas y con fuertes intereses económicos y de poder. 
Un tipo de capas tiene que ver con la construcción de discursos que diferencian y distancian los conocimientos "occidentales" o "eurocéntricos" de los de los habitantes locales, que son más "tradicionales" o "ancestrales". O que ni siquiera existen. En el caso de las Cinchona, tecnociencia y tecnocientíficos han soterrado a los sabedores que en algún momento (hasta nuestros días) han sido portadores de saberes y que luego han sido construidos como "otros" (en el sentido de Said, 2002). Al tiempo que indígenas o criollos han sido despojados de saberes y confianza en sus saberes, muchos se han vuelto adeptos a visiones más "occidentales" sobre cómo debe ser conocido, organizado, manipulado, apropiado, el mundo natural. Con ese desconocimiento del lugar de la autoridad se consigue imponer, con mayor intensidad, un modelo sobre quién conoce, quién debe conocer, qué es necesario saber y cómo hacerlo.

Al contribuir a apropiar (subjetiva y materialmente) las quinas, tecnociencia y colonialismo han acumulado por un lado y despojado por otro, localizando subjetiva y territorialmente las ganancias y las pérdidas, transformando modos preexistentes de actuar y pensar sobre la naturaleza. Han desplazado la autoridad hacia nuevos centros, semióticos y materiales, subalternizando a los sitios de sus portadores originales, nombrados como "periferias", negando autoridades previas y aún las vigentes.

Quizás el efecto más perverso de esos procesos es que una gran población lo haya creído, aunando a la construcción de subalternidades con vocablos que, a lo largo del tiempo, han cambiado desde "salvajes" y "atrasados" hasta "subdesarrollados" o "tercermundistas", entre otros.

\section{NOTAS}

1. Un documento producido como resultado de esas expediciones y conocido hace relativamente poco tiempo relata la búsqueda de quinas y otras plantas realizadas por Juan Tafalla (Estrella, 1989).

2. Banda C., Francisco (1943), "Quinine and substitutes". Records of the Reconstruction Finance Corporation (Record Group 234), Carpeta "Quinine and substitutes"; Caja 33, Quartz Crystal Processing Equipment-Quinine; Records of the Defense Supplies Corporation Commodity Procurement File 1942-49, Entry 159. National Archives at College Park.

3. "Agricultural Cooperation in the Americas" (1944), Geographical Review, 34 (1), pp. 151-153.

4. Ecuadorian Economic Resources Mission (1942), "Preliminary production plan and cost estimate for large scale rubber and abaca production in Ecuador under a national corporation". National Agriculture Library.
Reflexionar sobre las capas de tecnociencia y colonialismo reitera que el colonialismo y el imperialismo requieren de tecnociencia, y que en esas historias parecen cruciales también los silencios y ausencias, las que ocurren por la invisibilización de saberes y sabedores locales a lo largo de la historia, su desvalorización y soterramiento. Ayuda a entender en parte cómo se ha descentrado el lugar donde se conoce y el de autoridad, la desterritorialización de las producciones y el control la tecnología.

En el mundo contemporáneo también es posible detectar capas de tecnociencia y colonialismo, quizás no tanto en las quinas como en otras formas de apropiación de la naturaleza y de los saberes acerca de ella. Las actuaciones y actores tienen nuevos nombres y no necesariamente participan en instituciones que se llamen a sí mismas imperiales o coloniales, aunque sus actos parezcan revelar la larga duración del colonialismo y la colonialidad. Muchas emergen o reemergen en forma de actividades que hoy llamamos "extractivistas", "biopiratería", "mercantilización de la naturaleza", "exterminio de pueblos indígenas", entre otros.

De allí la necesidad de realizar continuos esfuerzos para pensar y actuar la significación, apropiación y transformación de la naturaleza, en la historia y en el presente. La historia de las capas de colonialismo alrededor de las quinas revela solo algunas taras que han existido para alcanzar sociedades libres, tanto en los espacios donde las externalidades negativas del colonialismo han sido más encarnadas, pero también desde donde ha sido más ejercido.
5. Reconstruction Finance Corporation (1948), "Contracts in Cinchona bark and quinine program, Defense Supplies Corporation". Records of the Reconstruction Finance Corporation (Record Group 234), Carpeta "General Commodities Cinchona"; Caja 5; Records of the United States Commercial Company, Administrative Subject File 1942-48, Entry 199. National Archives at College Park.

6. Reconstruction Finance Corporation (22 de febrero de 1943), "Conversation between representatives of the WPB and the BEW, Keith G. Cone, Daily Negotiations Report, Board of Economic Warfare, Office of Imports". Records of the Reconstruction Finance Corporation (Record Group 234), Carpeta "General Commodities Cinchona", Caja 5, Records of the United States Commercial Company, Administrative Subject File 1942-48, Entry 199. National Archives at College Park.

7. Camp, Wendell H. (1944-1945), "Notas diarias de Wendell Camp, Cuenca". Wendell Holmes (Red) Camp Papers, Car- 
peta "Ecuador Correspondence Personal Diary April 31944 - September 28 1945", Caja 1, Series 3, Field Trips and Expeditions. New York Botanical Garden.

8. Acosta Solís, Misael (1951), "Carta a Wendell H. Camp, 16 de marzo", y Camp, Wendell H. (1951), "Carta a Misael Acosta Solís, 14 de febrero". Wendell Holmes (Red) Camp Papers), Carpeta “Correspondence Acosta Solís", Caja 1, Series 2, Correspondence. New York Botanical Garden.

9. Wiggins, Ira (2 de noviembre de 1945), "Carta a William Steere". William C. Steere Papers, 1927-1958, Carpeta "Wiggins", Box 8, Series 8. New York Botanical Garden Archives.

10. Courand, Claude (Acting Special Representative) (4 de diciembre de 1944), "Letter to Rex A. Anderson, Assistant

\section{BIBLIOGRAFÍA}

Alimonda, Héctor (2011), “La colonialidad de la naturaleza. Una aproximación a la Ecología Política latinoamericana”. En: Alimonda, Héctor (coord.), La Naturaleza colonizada. Ecología política y minería en América Latina, Buenos Aires, Ediciones Ciccus y CLACSO, pp. 21-58.

Andersson, Lennart (1998), "A revision of the genus Cinchona (Rubiaceae-Cinchoneae)". En Memoirs of the New York Botanical Garden, vol. 80, pp. 1-75.

Balslev, Henrik, y Joyal, Elaine (1980), “Plant Collectors in Ecuador: Camp, Prieto, Jorgensen and Giler”, Brittonia, 32(4), pp. 437-451, [en línea], http://www.jstor.org/stable/2806148 [consultado el 18/1/2017].

Bleichmar, Daniela (2009), "Visible empire: scientific expeditions and visual culture in the Spanish Enlightenment", Postcolonial Studies, 12 (4), pp. 441-466, https://doi. org/10.1080/13688790903350674

Braudel, Fernand (1958/2007), "La larga duración, en la historia y las ciencias sociales", Revista Académica de Relaciones Internacionales, (5), pp. 1-56, [en línea], disponible en: http://www.relacionesinternacionales.info/ojs/article/ view/53/47.html [consultado el 30/11/2014].

Brockway, Lucile H. (1979), "Science and Colonial Expansion: The Role of the British Royal Botanic Gardens", American Ethnologist, 6 (3), pp. 449-465, https://doi.org/10.1525/ ae.1979.6.3.02a00030

Caldas, Francisco José de (1805/1966), “Memoria sobre el estado de las quinas en general y en particular sobre la de Loja". En: Obras completas de Francisco José de Caldas: publicadas por la Universidad Nacional de Colombia como homenaje con motivo del sesquicentenario de su muerte 1816 - Octubre 29 -1966, Bogotá, Imprenta Nacional, pp. 241-260.

Camp, Wendell H. (1949), "Cinchona at High Altitudes in Ecuador", Brittonia, 6 (4), pp. 394-430, [en línea], disponible en: http:// www.jstor.org/stable/2804925 [consultado el 24/02/2016].
Administrator, Office of Management Services". Records of the Reconstruction Finance Corporation (Record Group 234), Carpeta "Ecuador General October 1944 thru", Caja 11, Ecuador October 1944 to Finland, Records of the United States Commercial Company General Country File 1942-48, Entry 200. National Archives at College Park.

11. Vandercook, E.V. (Sin fecha), "Letter to Mr. de Camp". Records of the Reconstruction Finance Corporation (Record Group 234), Carpeta "Ecuador-General"; Caja 10, China 1945-Ecuador September 1944, Records of the United States Commercial Company General Country File 1942-48, Entry 200. National Archives at College Park.

Camp, Wendell H. (1952), "Plant hunting in Ecuador", Memoirs of the New York Botanical Garden, 8 (1), pp. 1-24.

Cañizares-Esguerra, Jorge (2006), Nature, Empire, and Nation. Explorations of the History of Science in the Iberian World, Stanford, Stanford University Press.

Castro-Gómez, Santiago (2005), La hybris del punto cero: ciencia, raza e ilustración en la Nueva Granada, Bogotá, Pontificia Universidad Javeriana.

Castro-Gómez, Santiago, y Grosfoguel, Ramón (eds.) (2007), El giro decolonial: reflexiones para una diversidad epistémica más allá del capitalismo global, Bogotá: Siglo del Hombre Editores, Universidad Central -Instituto de Estudios Sociales Contemporáneos- y Pontificia Universidad Javeriana -Instituto Pensar-.

Crawford, Matthew James (2007), “"Para desterrar las dudas y adulteraciones": Scientific Expertise and the Attempts to Make a Better Bark for the Royal Monopoly of Quina (17511790)", Journal of Spanish Cultural Studies, 8 (2), pp. 193212, https://doi.org/10.1080/14636200701431016

Cueto, Marcos (ed.) (1995), Saberes Andinos: Ciencia y Tecnología en Bolivia, Ecuador y Perú, Lima, Instituto de Estudios Peruanos.

Cuvi, Nicolás (2009), Ciencia e imperialismo en América Latina: la Misión de Cinchona y las estaciones experimentales agrícolas, Tesis de Doctorado en Historia de la Ciencia, Barcelona, Universitat Autònoma de Barcelona, [en línea], http:// hdl.handle.net/10803/5182 [consultado el 30/3/2017].

Cuvi, Nicolás (2011), "The Cinchona Program (1940-1945): science and imperialism in the exploitation of a medicinal plant", Dynamis, 31 (1), pp. 183-206, [en línea], disponible en: http://www.raco.cat/index.php/Dynamis/article/ view/248584 [consultado el 25/6/2012].

Cuvi, Nicolás (2012), "Big Science and the Enchantment of Growth in Latin America", Global Environment, A Journal of History and Natural and Social Sciences, 10, pp. 16-41. 
de Andrés Turrión, María Luisa (1989), “Quina del Nuevo Mundo para la Corona española", Asclepio: archivo iberoamericano de historia de la medicina y antropología médica, 41 (1), pp. 305-324

de La Condamine, Charles Marie (1745/1986), Viaje a la América Meridional por el Río de las Amazonas, BarceIona, Alta Fulla.

de La Condamine, Charles Marie (1778/1986), Estudio sobre la quina, Barcelona, Alta Fulla.

Drayton, Richard Harry (2000), Nature's government: science, imperial Britain, and the 'Improvement' of the World, New Haven, Yale University Press.

Espejo, Eugenio (1792), Memorias sobre el corte de quinas y Voto de un Ministro Togado. Quito.

Estrella, Eduardo (1989), “Introducción histórica: La Expedición de Juan Tafalla a la Real Audiencia de Quito (1799-1808) y la 'Flora Huayaquilensis', de Juan José Tafalla Navascués". En: Estrella, Eduardo (ed.), Flora Huayaquilensis .... I-CVI. (Vol. 1), Madrid, ICONA y CSIC.

Fernández Prieto, Leida (2013), "Islands of Knowledge: Science and Agriculture in the History of Latin America and the Caribbean", Isis, 104 (4), pp: 788-797, https://doi. org/10.1086/674945

Fernández Prieto, Leida (2015a), “Introducción. Ciencia, agricultura y saberes locales en América latina y el Caribe: nuevas perspectivas", Asclepio. Revista de Historia de la Medicina y de la Ciencia, 67 (1): p075, https://doi.org/10.3989/asclepio. 2015.01

Fernández Prieto, Leida (2015b), "Saberes Híbridos: las Sugar Companys y la moderna plantación azucarera en Cuba", Asclepio, 67 (1): p080, https://doi.org/10.3989/asclepio.2015.06

Gorbach, Frida, y López Beltrán, Carlos (eds.) (2008), Saberes locales. Ensayos sobre historia de la ciencia en América Latina. Zamora, El Colegio de Michoacán.

Gramiccia, Gabriele (1988), The life of Charles Ledger (18181905), Alpacas and quinine, Londres, Macmillan.

Guerra, Francisco (1977), "The introduction of Cinchona in the treatment of malaria", Pts. I y II, Journal of Tropical Medicine and Hygiene, 80(6), pp.112-118, 135-140.

Haggis, A. W (1941), Fundamental errors in the early history of cinchona, Bulletin of the History of Medicine, 10, pp. 586-592.

Headrick, Daniel R. (1989), Los instrumentos del imperio. Tecnología e imperialismo europeo en el siglo XIX, Madrid, Alianza Editorial.

Henningson, Berton E. (1981), United States. Office of Foreign Agricultural Relations. United States agricultural trade and development policy during World War II: the role of the Office of Foreign Agricultural Relations, Ph.D. thesis, University of Arkansas.

Herrera, Alexander, y Ali, Maurizio (2009), "Paisajes del desarroIlo: la ecología de las tecnologías andinas", Antípoda. Revista de Antropología y Arqueología (8), pp. 169-194, [en línea], http://www.scielo.org.co/scielo.php?script=sci_arttext\&pi $d=$ S1900-54072009000100008 [consultado el 10/3/2017].

Hodge, Walter (1948), "Wartime Cinchona Procurement in Latin America", Economic Botany, 2 (3), pp. 229-257.

Humboldt, Alexander von. (1821), "Account of the Cinchona Forests of South America". En: An illustration of the genus Cinchona : comprising descriptions of all the officinal Peruvian barks, including several new species, Baron de Humboldt's account of the Cinchona forests of South Ameri$c a$, and Laubert's Memoir on the different species of quinquina : to which are added several dissertations of Don Hippolito Ruiz on various medicinal plants of South America ... And A short account of the spikenard of the ancients, pp. 19-59, Londres, Printed for J. Searle.

Jarcho, Saul (1993), Quinine's Predecessor: Francesco Torti And The Early History Of Cinchona, Baltimore, Johns Hopkins University Press.

Jussieu, Joseph (1737/1936), Description de l'arbre à Quinquina, París, La Société du Traitement des Quinquines, M. Pancier Editeur.

Krige, John (2006), American hegemony and the postwar reconstruction of science in Europe, Cambridge, Mass., MIT Press.

López Piñero, José María, y Pardo Tomás, José (1994), “Nuevos materiales y noticias sobre la Historia de las Plantas de Nueva España, de Francisco Hernández", Cuadernos Valencianos de Historia de la Medicina y de la Ciencia, 44. Valencia, Instituto de Estudios Documentales e Históricos sobre la Ciencia, Universitat de València-CSIC.

MacLeod, Roy (2000), "Introduction", Osiris, 15, pp. 1-13, [en línea], disponible en: http://www.jstor.org/stable/301937 [consultado el 23/9/2014].

Markham, Clements R. (1862), Travels in Peru and India while Superintending the Collection of Cinchona Plants and Seeds in South America and their Introduction into India, Londres, John Murray.

Mutis, José Celestino (1828/1994), El Arcano de la Quina, Madrid, Fundación de Ciencias de la Salud.

Nieto Olarte, Mauricio (2006), Remedios para el imperio: historia natural y la apropiación del Nuevo Mundo (2 ed.), Bogotá, Universidad de los Andes.

Ortiz Crespo, Fernando (1994), "La Cinchona antes y después del Virreinato del Conde de Chinchón”, Interciencia, 19 (3), pp. 130-136. 
Osborne, Michael A. (2000), "Acclimatizing the World: A History of the Paradigmatic Colonial Science", Osiris, 15, pp. 135-151, [en línea], disponible en: http://www.jstor.org/ stable/301945, [consultado el 30/6/2015].

Palladino, Paolo y Worboys, Michael (1993), "Science and Imperialism", Isis, 84 (1), pp. 91-102.

Pardo Valle, Nazario (1947), Legislación boliviana sobre quinas, quinina y lucha anti-palúdica, La Paz, Editorial Don Bosco.

Pelletier, Pierre Joseph y Caventou, Joseph B. (1821), Analyse chimique des quinquina: suivie d'observations médicales sur l'emploi de la quinine et de la chinchonine, Paris, Colas.

Philip, Kavita (1995), "Imperial Science Rescues a Tree: Global Botanic Networks, Local Knowledge and the Transcontinental Transplantation of Cinchona", Environment and History, 1 (2), pp. 173-200, [en línea], disponible en: http://www.jstor. org/stable/20722975 [consultado el 20/7/2015].

Quijano, Aníbal (2000), "Colonialidad del poder, eurocentrismo y América Latina". En: Lander, Edgardo (comp.), La colonialidad del saber: eurocentrismo y ciencias sociales. Perspectivas Latinoamericanas, Buenos Aires, CLACSO (Consejo Latinoamericano de Ciencias Sociales), pp. 201-246.

Raj, Kapil (2007), Relocating modern science: circulation and the construction of knowledge in South Asia and Europe, 16501900, Houndmills, Basingstoke, Hampshire England, y Nueva York: Palgrave Macmillan.

Rosengarten, Frederic (1944), History of the Cinchona project of Merck \& co., inc. and Experimental plantations, inc., 19341943, Rahway, New Jersey, Merck \& co.

Ruiz, Hipólito (1792/1994), Quinología. Suplemento a la Quinología, Madrid, Fundación Ciencias de la Salud.

Said, Edward (2002), Orientalismo, Madrid, Debate.

Seth, Suman (2009), "Putting knowledge in its place: science, coIonialism, and the postcolonial", Postcolonial Studies, 12 (4), pp. 373-388, https://doi.org/10.1080/13688790903350633

Spruce, Richard (1859/1996). "Notas de una visita a los bosques de cinchona en el valle de Alausí, en las estribaciones occidentales de los Andes de la región de Quito. Carta a William Hooker". En: Wallace, Alfred R. (ed.), Notas de un botánico en el Amazonas y en los Andes. Apuntes de los viajes por el Amazonas y sus tributarios, El Trombetas, Río Negro, Uaupés, Casiquiari, Pacimoni, Huallaga y Pastaza; también por las cataratas del Orinoco, a lo largo de la Cordillera de los Andes ecuatorianos y peruanos y por las costas del Pacífico, durante los años 1849-1864, Quito, Abya-Yala, pp. 515-534.

Spruce, Richard (1860/1996). "Los bosques de cascarilla de "EI Limón" en las estribaciones occidentales del Chimborazo". En: Wallace, Alfred R. (ed.), Notas de un botánico en el Amazonas y en los Andes. Apuntes de los viajes por el Amazonas y sus tributarios, El Trombetas, Río Negro, Uaupés, Casiquiari, Pacimoni, Huallaga y Pastaza; también por las cataratas del Orinoco, a lo largo de la Cordillera de los Andes ecuatorianos y peruanos y por las costas del Pacífico, durante los años 1849-1864, Quito, Abya-Yala, pp. 535-580.

Stork, Gilbert; Niu, Deqiang; Fujimoto, A.; Koft, Emil R.; Balkovec, James M.; Tata, James R. y Dake, Gregory R. (2001), "The First Stereoselective Total Synthesis of Quinine", Journal of the American Chemical Society, 123(14): pp. 32393242, https://doi.org/10.1021/ja004325r

Tucker, Richard P. (2000), Insatiable appetite: the United States and the ecological degradation of the tropical world, Berkeley, University of California Press.

Urteaga González, Luis (1987), La tierra esquilmada: las ideas sobre la conservación de la naturaleza en la cultura española del siglo XVIII, Madrid, Consejo Superior de Investigaciones Científicas.

Wellcome Historical Medical Museum (1930), Souvenir: Cinchona Tercentenary Celebration and exhibition at the Wellcome Historical Medical Museum, Londres, Wellcome Foundation.

Wiselogle, Frederick Y. (1946), A Survey on Antimalarial Drugs 1941-1945 (2 vols), Ann. Arbor, Michigan, J.W. Edwards.

Woodward, Robert D. y Doering, William E. (1944), "The total synthesis of quinine", Journal of the American Chemical Society, 66 (5), pp. 849-849, https://doi.org/10.1021/ ja01233a516

Worrall, Eve, Basu, Suprotik, y Hanson, Kara (2005), "Is malaria a disease of poverty? A review of the literature", Tropical Medicine \& International Health, 10 (10), pp. 1047-1059, https://doi.org/10.1111/j.1365-3156.2005.01476.x 